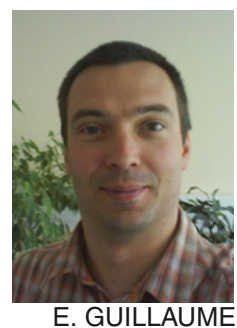

\title{
Incertitude sur la mesure par un « cône calorimètre » du dégagement de chaleur produit lors de la combustion d'un matériau
}

\section{Uncertainty on heat release rate measurement with cone calorimeter during the combustion of a material}

\author{
Eric GUILLAUME, Damien MARQUIS, Laurent SARAGOZA et Catherine YARDIN
}

Laboratoire national de métrologie et d'essais (LNE), 29 Avenue Roger Hennequin, 78197 Trappes Cedex, France, eric.guillaume@lne.fr.

\begin{abstract}
Résumé
Le «cône calorimètre » est un appareil de base pour les essais de comportement au feu des matériaux. Il sert à mesurer le dégagement de chaleur, communément appelé dans ce domaine « débit calorifique », des matériaux soumis à une densité de flux de chaleur par une méthode thermochimique. Or cette méthode est complexe et fait appel à de nombreux paramètres. La méthode est aujourd'hui normalisée et certains des paramètres mesurés servent à classer les matériaux selon différentes réglementations. La détermination de l'incertitude de mesure de l'équipement est ainsi primordiale. L'article présente le calcul de l'incertitude appliqué à cet équipement, ainsi qu'un exemple d'application à une série d'essais de comportement au feu d'un matériau.
\end{abstract}

MOTS CLÉS : CALORIMÉTRIE， THERMOCHIMIE， CONE CALORIMÈTRE, INCERTITUDE, MATÉRIAU, FEU.

\footnotetext{
Abstract

Cone calorimeter is one of the basic test bench for fire behaviour tests on materials. It is used to measure heat release rate of materials under heat flux by thermochemical method. This method is complex and uses a large number of parameters. Test method is nowadays standardized and some of measured parameters are used for material ranking in some regulations. It is therefore essential to determine test bench uncertainty. This paper presents uncertainty calculation applied to the test method and an example of it application to a set of fire behaviour tests on a material.
}

\section{Contexte}

Le «cône calorimètre » est l'un des moyens d'essai de base dans le domaine du comportement au feu. Cet essai a été développé par Babrauskas dans les années 1980 [1,2], afin d'étudier le dégagement de chaleur produit lors de la combustion de matériaux soumis à une densité de flux radiatif incident. La quantité de chaleur produite par unité de temps est mesurée par calorimétrie à consommation d'oxygène [3,4], selon la théorie de Thornton [5].

Le banc d'essai est aujourd'hui normalisé selon la norme ISO 5660-1 [6] et commence à être utilisé dans différentes réglementations, en particulier dans les transports publics de passagers.

Dans cette norme, les dimensions des échantillons caractérisés ont une surface de $100 \mathrm{~mm} \times 100 \mathrm{~mm}$ et une épaisseur maximum de $50 \mathrm{~mm}$. Les échantillons sont placés sous un four de forme conique rayonnant un flux incident prédéterminé.

Le dégagement de chaleur de cette éprouvette est déterminé par la méthode de mesure de la consommation d'oxygène (sans correction de $\mathrm{CO}$ et $\mathrm{CO}_{2}$ ) Les paramètres déterminés dans le calcul d'incertitude sont :

- le dégagement de chaleur instantané $\dot{q}(t)$ et le dégagement de chaleur maximum $\dot{q}_{\max }$; 


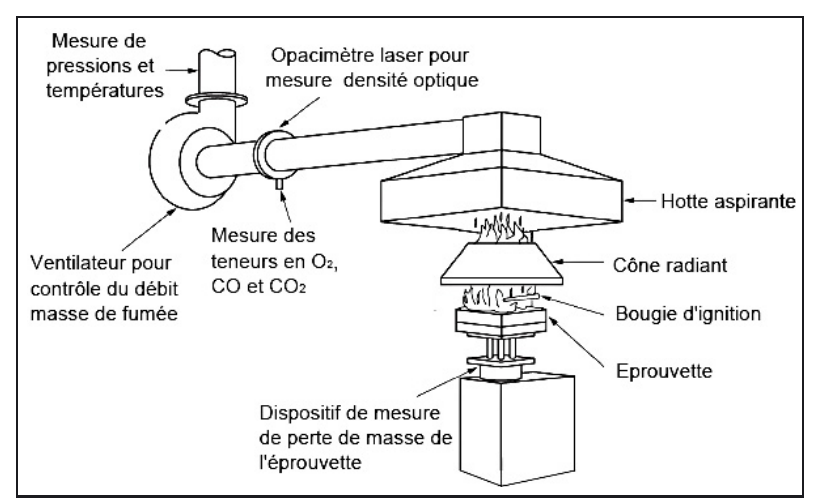

Fig. 1. - Schéma du banc de mesure de type «cône calorimètre ».

- le dégagement total de chaleur $\dot{Q}$ correspondant à l'intégrale de la courbe de dégagement de chaleur en fonction du temps ;

- d'autres paramètres calculés d'après la courbe de débit calorifique, comme le MARHE, paramètre de classement des matériaux dans le domaine ferroviaire.

\section{Détermination du dégagement de chaleur}

\subsection{Principe de mesure}

Dans le secteur du comportement au feu des matériaux, les méthodes de détermination du dégagement de chaleur sont en général basées sur des principes thermochimiques plutôt que calorimétriques. En effet, les rapports entre les énergies libérées sous forme de rayonnement et de convection, ainsi que les pertes thermiques, sont difficiles à estimer au regard de la variété des combustibles et des conditions de combustion rencontrées. De ce fait, les méthodes thermiques demeurent moins précises que celles fondées sur l'analyse chimique des fumées.

Les mesures de calorimétrie instantanée au «cône calorimètre » sont basées sur la mesure de la diminution d'oxygène $[3,4]$ : une éprouvette est soumise à une source rayonnante et la combustion de cette éprouvette, qui en résulte, consomme du dioxygène. La diminution de la teneur en dioxygène dans les fumées peut ensuite être reliée à la quantité de chaleur produite par la combustion. Un schéma du banc de mesure est présenté dans la figure 1.

Pour la plupart des matériaux, Thornton [5] a constaté expérimentalement que la consommation de $1 \mathrm{~kg}$ de dioxygène correspond généralement à une énergie libérée par combustion de 13,1 MJ.

Le dégagement de chaleur instantané $\dot{q}(t)$, fonction du temps $t$ s'exprime en fonction de la consommation de l'oxygène de la façon suivante :

$$
\dot{q}(t)=E \cdot\left(x_{\mathrm{a}}-x_{\mathrm{b}}(t)\right) \cdot \frac{V_{\mathrm{s}}(t)}{e}
$$

où :

- $E$ est le facteur de conversion énergétique de Thornton, valant en moyenne 419,2 kJ par mole de dioxygène $\left(\mathrm{O}_{2}\right)$ consommé ;

- $x_{\mathrm{a}}$ est la fraction molaire de dioxygène dans l'air ambiant (en général 20,95\%);

$-x_{\mathrm{b}}(t)$ est la fraction molaire de dioxygène dans les fumées mesurée en fonction du temps $t$;

- $V_{\mathrm{s}}(t)$ est le débit molaire des fumées (en mol/s d'air);

- $e$ est le facteur d'expansion de l'air dont l'oxygène a été consommé et vaut environ 1,105.

\subsection{Formule de calcul}

Le « débit calorifique », exprimé en watts, est donné dans la norme ISO 5660 par les formules (2) et (3) :

$$
\dot{q}(t)=E \times 1,10 \times \dot{m}_{\mathrm{e}} \times \frac{x_{\mathrm{O}_{2}}^{0}-x_{\mathrm{O}_{2}}}{1,105-1,5 x_{\mathrm{O}_{2}}}
$$

où

$$
\dot{m}_{\mathrm{e}}=C \sqrt{\frac{\Delta P}{T_{\mathrm{e}}}}
$$

avec :

- $E$ : facteur de Thornton (en $\mathrm{J} \cdot \mathrm{kg}^{-1}$ );

- $\dot{m}_{\mathrm{e}}$ : débit massique des effluents au niveau du diaphragme $\left(\mathrm{en} \mathrm{kg} \cdot \mathrm{s}^{-1}\right)$;

- $C$ : coefficient d'étalonnage du diaphragme servant de débitmètre $\left(\mathrm{en}^{1 / 2} \cdot \mathrm{kg}^{1 / 2} \cdot \mathrm{K}^{1 / 2}\right)$;

- $\Delta P$ : différence de pression mesurée de chaque côté du diaphragme à chaque instant, exprimée en pascal ;

- $T_{\mathrm{e}}$ : température des fumées au niveau du diaphragme $($ en $\mathrm{K})$;

$-x_{\mathrm{O}_{2}}^{0}$ : fraction molaire initiale en oxygène, mesurée durant une période d'une minute ;

- $x_{\mathrm{O}_{2}}$ : fraction molaire en oxygène, mesurée à chaque instant et décalée du temps de réponse de l'analyseur.

Le facteur 1,105 correspond au facteur d'expansion du gaz, c'est-à-dire au fait qu'il y ait plus de moles de gaz après combustion qu'avant. Il est lié à la production estimée d'eau par le feu et éliminée par le séchage. Le coefficient 1,5 est une estimation de la production d'eau et de $\mathrm{CO}_{2}$ par rapport à la consommation d'oxygène. Le facteur 1,10 est une estimation du rapport des masses molaires de l'air et de l'oxygène.

Le facteur de Thornton exprime l'enthalpie de combustion relative à la consommation d'oxygène. Par défaut, dans le cas d'essais réglementaires, sa valeur est fixée à $13,1 \mathrm{MJ} \cdot \mathrm{kg}^{-1}$, sans incertitude. Le dégagement de chaleur mesuré est alors une valeur conventionnelle 
Tableau 1

Termes sources d'erreur identifiés pour la mesure du dégagement de chaleur.

\begin{tabular}{|l|l|c|c|}
\hline \multicolumn{2}{|c|}{ Facteur } & $\begin{array}{c}\text { Nature } \\
\text { de l'erreur }\end{array}$ & Commentaire \\
\hline \multirow{3}{*}{$\begin{array}{l}\text { Débit des } \\
\text { effluents }\end{array}$} & Facteur $C$ & Justesse, fidélité & Pris en compte dans le calcul \\
\cline { 2 - 4 } & $\Delta P$ & Justesse, fidélité & $\begin{array}{c}\text { Pris en compte dans le calcul - Justesse corrigée par un } \\
\text { polynôme }\end{array}$ \\
\cline { 2 - 4 } & Température $\left(T_{\mathrm{e}}\right)$ & Justesse, fidélité & Pris en compte dans le calcul \\
\hline Facteur de Thornton & Justesse & $\begin{array}{c}\text { Vaut 13,1 MJ·kg }{ }^{-1} \text { par défaut - Non pris en compte dans } \\
\text { le calcul (calcul conventionnel) }\end{array}$ \\
\hline Facteur d'expansion & Justesse & $\begin{array}{c}\text { Vaut 1,105 par défaut } \text { - Non pris en compte dans } \\
\text { le calcul (calcul conventionnel) }\end{array}$ \\
\hline $\begin{array}{l}\text { Rapport des densités de l'air et de } \\
\text { l'oxygène }\end{array}$ & Justesse & $\begin{array}{c}\text { Vaut 1,10 par défaut }- \text { Non pris en compte dans le calcul } \\
\text { (calcul conventionnel) }\end{array}$ \\
\hline \multirow{2}{*}{ Analyse des gaz } & $x_{\mathrm{O}_{2}}^{0}$ & Justesse, fidélité & Pris en compte dans le calcul \\
\cline { 2 - 4 } & $x_{\mathrm{O}_{2}}$ instantané & Justesse, fidélité & Pris en compte dans le calcul \\
\hline
\end{tabular}

et le biais n'est pas connu. Si l'essai est effectué dans le cadre d'une étude de matériau, l'amélioration de la justesse peut être souhaitable, et un facteur de Thornton plus spécifique peut alors être utilisé. Dans ce cas, ce facteur est assorti d'une incertitude.

Dans la mesure du dégagement de chaleur, la valeur vraie ne peut être estimée que pour les essais sur des combustions de gaz étalons. Pour les combustions de matériaux solides, elle est inaccessible et le biais peut être important. Pour des essais réglementaires, il convient néanmoins de comparer les mesures de différents laboratoires effectuées dans les mêmes conditions, d'où l'utilisation de la formule simplifiée et d'un facteur de Thornton fixé à $13,1 \mathrm{MJ} \cdot \mathrm{kg}^{-1}$.

La démonstration de cette équation est donnée dans les références $[3,4,7]$. Le résultat est en général exprimé ramené par unité de surface exposée à l'éclairement énergétique.

\subsection{Origine et nature des erreurs et des incertitudes}

Le principe de détermination du dégagement de chaleur par la mesure de la consommation d'oxygène implique qu'une énergie dégagée soit associée à la quantité d'oxygène consommée par le feu. Cette théorie est vérifiée partiellement pour de nombreux matériaux; les écarts proviennent principalement de la présence d'atomes autres que le carbone et l'hydrogène dans le combustible, ainsi que du caractère incomplet de la combustion. Certaines corrections sont possibles afin de prendre en compte les transferts d'énergie associés à une oxydation partielle.

Ainsi, dans le cas de la formule considérant la consommation d'oxygène seule, l'incertitude calculée est une incertitude liée principalement à la répétabilité et à la reproductibilité. La justesse n'est pas totalement prise en compte dans le calcul, car le facteur de Thornton et le facteur d'expansion des gaz sont supposés constants et parfaitement connus.
Cette formule simplifiée donne donc la fidélité la plus faible, mais le biais peut être important. Des formules plus élaborées ont été établies. Dans ces dernières, le biais est diminué par adjonction de termes correctifs, mais la présence de facteurs supplémentaires entraîne une dégradation de la fidélité.

Or, le dégagement de chaleur est fréquemment utilisé à des fins comparatives et de classification des matériaux. Ainsi, la justesse n'est pas primordiale dans la plupart des référentiels. Seule compte la comparaison des résultats dans les mêmes conditions. En somme, dans cette étude, nous étudions des conditions normalisées et le biais est toujours présent mais non quantifié.

Le tableau 1 reprend les différents termes sources de l'erreur de la formule simplifiée de mesure de dégagement de chaleur et la nature de l'erreur associée. Différentes déterminations de l'incertitude de mesure sur la méthode ont été publiées, en particulier par Enright et Fleischmann [8], Zhao puis Zhao et Dembsey [9-11]. Ces déterminations ont mis en évidence des termes sources d'erreur similaires.

\section{Résultats d'essais interlaboratoires}

\subsection{Données de la norme ISO 5660-1}

La répétabilité et la reproductibilité de l'essai au « cône calorimètre » ont été déterminées grâce à deux études : l'une de l'ISO TC92/SC1/WG5 et l'autre de l'ASTM E05 SC21 TG60. Elles sont reprises dans la norme ISO 5660-1 :2002 et dans le tableau 2 pour les paramètres principaux (données à $95 \%$ ), pour des matériaux ayant un comportement classique ainsi que pour des matériaux ayant un comportement intumescent. Les matériaux intumescents présentent une répétabilité et une reproductibilité nettement supérieures, probablement dues fait de leur comportement plus complexe. Cela tend à démontrer que l'effet lié au matériau sera l'un des facteurs prépondérants sur l'estimation de l'incertitude. 
Tableau 2

Répétabilité et reproductibilité issues d'une comparaison interlaboratoire [6].

\begin{tabular}{|c|c|c|c|}
\hline Paramètre & Domaine & Répétabilité & Reproductibilité \\
\hline $\begin{array}{c}\text { Temps d'inflammation } \\
t_{\mathrm{ig}}(\mathrm{en} \mathrm{s})\end{array}$ & 5 à 150 & $4,1+0,125 t_{\mathrm{ig}}$ & $7,4+0,2 t_{\mathrm{ig}}$ \\
\hline $\begin{array}{c}\text { Dégagement de chaleur } \\
\text { surfacique maximal } \\
\dot{q}_{\max }^{\prime \prime}\left(\mathrm{en} \mathrm{kW} \cdot \mathrm{m}^{-2}\right)\end{array}$ & $\begin{array}{c}70 \text { à } 1120 \\
\text { (matériaux classiques) }\end{array}$ & $13,3+0,131 \dot{q}_{\max }^{\prime \prime}$ & $60,4+0,141 \dot{q}_{\max }^{\prime \prime}$ \\
\cline { 2 - 4 } (matériaux intumescents) & 83 à 55 & $36,6+0,064 \dot{q}_{\max }^{\prime \prime}$ & $36,6+0,33 \dot{q}_{\max }^{\prime \prime}$ \\
\hline $\begin{array}{c}\text { Chaleur totale surfacique } \\
\text { dégagée } \dot{Q}^{\prime \prime}\left(\mathrm{en} \mathrm{MJ} \cdot \mathrm{m}^{-2}\right)\end{array}$ & 5 à 720 & $7,4+0,068 \dot{Q}^{\prime \prime}$ & $11,8+0,088 \dot{Q}^{\prime \prime}$ \\
\hline
\end{tabular}

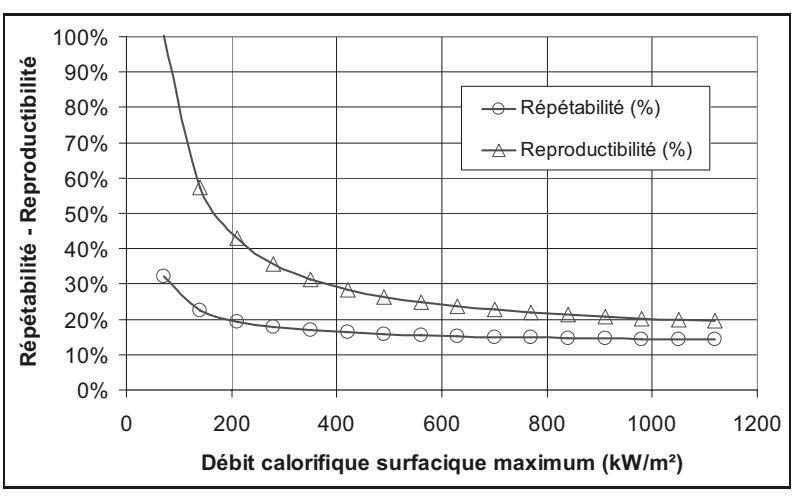

Fig. 2. - Répétabilité et reproductibilité de la méthode de mesure du dégagement de chaleur selon la norme ISO 5660-1 [6] pour des matériaux classiques.

Les répétabilités et reproductibilités de la mesure du dégagement de chaleur maximum sont représentées en figure 2. De plus, les essais réalisés dans ce cadre ont montré que le temps de réponse de la mesure était dépendant du temps de réponse de l'analyseur d'oxygène. L'incertitude sur le facteur $C$ semble expliquer la reproductibilité.

\subsection{Résultats de mesure obtenus pour l'établissement de la norme STANAG 4602}

La norme OTAN STANAG 4602 [12] reprend la méthode ISO 5660-1 dans son document d'application AFAP-5 [13]. Lors de l'établissement de cette norme, une campagne d'essais interlaboratoires a été réalisée. Elle a porté sur deux matériaux (EPDM et PMMA) testés à trois niveaux de densité de flux incident (source de rayonnement à laquelle est soumis le matériau).

Les données de répétabilité $(r)$ et de reproductibilité $(R)$ obtenues pour le dégagement de chaleur surfacique maximum $\left(\dot{q}_{\max }^{\prime \prime}\right)$ et la chaleur totale surfacique $\left(\dot{Q}^{\prime \prime}\right)$ sur ces deux matériaux sont reprises dans le tableau 3 après recherche de valeurs aberrantes. Ces résultats montrent des répétabilités satisfaisantes, hormis pour l'essai avec un éclairement énergétique de $35 \mathrm{~kW} \cdot \mathrm{m}^{-2}$ sur l'EPDM, du fait de la dispersion d'un laboratoire.
Tableau 3

Répétabilité $(r)$ et reproductibilité $(R)$ pour $N$ laboratoires issues d'une comparaison interlaboratoire [13].

\begin{tabular}{|c|c|c|c|c|c|}
\hline Matériau & $\begin{array}{c}\text { Densité } \\
\text { de flux } \\
\text { incident } \\
\left(\mathbf{k W} \cdot \mathbf{m}^{-2}\right)\end{array}$ & $N$ & $\begin{array}{c}\dot{q}_{\max }^{\prime \prime} \\
\left(\mathbf{k W} \cdot \mathbf{m}^{-2}\right)\end{array}$ & $\begin{array}{c}r \\
(\%)\end{array}$ & $\begin{array}{c}R \\
(\%)\end{array}$ \\
\hline \multirow{3}{*}{ EPDM } & 35 & 3 & 170,8 & 24,1 & 35,5 \\
\hline & 50 & 2 & 205,8 & 10,3 & 12,6 \\
\hline & 75 & 3 & 234,2 & 15,6 & 15,6 \\
\hline \multirow{3}{*}{ PMMA } & 35 & 6 & 724,5 & 15,3 & 29,7 \\
\hline & 50 & 6 & 890,1 & 12,3 & 26,5 \\
\hline & 75 & 4 & 1174,2 & 20,3 & 64,6 \\
\hline & & & & & \\
\hline Matériau & $\begin{array}{c}\text { Densité } \\
\text { de flux } \\
\text { incident } \\
\left(\mathbf{k W} \cdot \mathbf{m}^{-2}\right)\end{array}$ & $N$ & $\begin{array}{c}\dot{Q}^{\prime \prime} \\
\left(\mathbf{M J} \cdot \mathbf{m}^{-2}\right)\end{array}$ & $\begin{array}{c}r \\
(\%)\end{array}$ & $\begin{array}{c}R \\
(\%)\end{array}$ \\
\hline \multirow{3}{*}{ EPDM } & 35 & 3 & 67,9 & 58,3 & 81,2 \\
\hline & 50 & 2 & 58,2 & 8,9 & 20,0 \\
\hline & 75 & 2 & 58,4 & 4,1 & 12,3 \\
\hline \multirow{3}{*}{ PMMA } & 35 & 6 & 616,0 & 8,8 & 14,6 \\
\hline & 50 & 6 & 621,3 & 13,2 & 25,1 \\
\hline & 75 & 4 & 592,0 & 14,2 & 37,7 \\
\hline
\end{tabular}

Globalement, les reproductibilités sont satisfaisantes, sauf dans le cas cité précédemment et dans le cas du dégagement de chaleur maximum pour le PMMA exposé à un éclairement énergétique de $75 \mathrm{~kW} \cdot \mathrm{m}^{-2}$, du fait d'une valeur isolée non aberrante d'un laboratoire. Lors de l'essai sur le PMMA à $75 \mathrm{~kW} \cdot \mathrm{m}^{-2}$, si ce laboratoire est retiré, les résultats présentent une moyenne de $1290 \mathrm{~kW} \cdot \mathrm{m}^{-2}$, une répétabilité de $20,4 \%$ et une reproductibilité de $36,4 \%$.

Ces valeurs sont comparables, à celles décrites précédemment et provenant de la norme, voire meilleures.

\section{4. Évaluation de l'incertitude sur la mesure du dégagement de chaleur}

\subsection{Principe du calcul de l'incertitude}

La méthode de calcul retenue est la propagation des incertitudes telle que décrite dans le GUM [14]. 


\subsubsection{Sources d'incertitude}

\subsubsection{Matériau}

L'une des principales sources d'incertitude est la composition même du matériau testé. L'hétérogénéité dans la réalisation des polymères ou dans la nature des matériaux naturels peut engendrer une incertitude. Ce phénomène, dénommé "effet matériau » est souvent considérable dans les essais au feu et dépend du matériau en essai. C'est pourquoi il est intégré dans la répétabilité des essais, sans pour autant que sa contribution puisse être clairement identifiée. Il est principalement une source d'incertitude de type aléatoire non maîtrisée.

\subsubsection{Dégradation thermique du matériau}

L'incertitude sur la dégradation thermique a deux origines : la difficulté d'évaluation du flux thermique incident et de positionner précisément l'allumage des gaz.

Premièrement, l'éprouvette est soumise à un éclairement énergétique issu d'un four radiant. La densité de flux surfacique est de $35 \mathrm{~kW} \cdot \mathrm{m}^{-2}$ ou $50 \mathrm{~kW} \cdot \mathrm{m}^{-2}$ selon le matériau testé, et est vérifiée à l'aide d'un fluxmètre. Une incertitude de réglage du four demeure. L'émissivité spectrale du four varie en fonction de son usure et de sa température. La transmission de la chaleur est censée être surtout de type radiatif, mais un transfert convectif subsiste, ne serait-ce qu'à la surface chauffée du fluxmètre. Les deux sont indissociables lors de l'étalonnage. De plus, l'émissivité de l'éprouvette varie en fonction de son état de surface, de l'humidité ambiante et de son conditionnement. L'éclairement énergétique de consigne est donné pour un corps noir, ce qui n'est pas le cas de l'éprouvette.

Deuxièmement, une incertitude demeure du fait de la position de la bougie d'allumage. Cette bougie, placée au-dessus du centre de l'éprouvette, peut ne pas se trouver dans le flux des gaz de pyrolyse concentrés et ne pas enflammer ceux-ci au moment souhaité. Or, l'inflammation et le flux radiatif issu de la flamme vers l'éprouvette ont un effet thermique considérable.

Ces différentes considérations sont de nature à générer un facteur d'incertitude difficilement évaluable.

\subsubsection{Mesure des concentrations en gaz et du débit}

Les paramètres mesurés utilisés dans la formule (2) de détermination du dégagement de chaleur sont la teneur en oxygène et le débit volumique des fumées. L'ensemble de ces mesures sont assorties d'une incertitude qui a vraisemblablement plusieurs origines : linéarité, répétabilité et dérive des analyseurs et du capteur de pression, ainsi que l'écart et l'amortissement du au circuit pneumatique de transport des gaz entre le point de prélèvement et le point d'analyse. Les termes correspondants sont ceux permettant le calcul du dégagement de chaleur instantané, et donc de l'incertitude associée.
Tableau 4

Étalonnage du capteur de pression.

\begin{tabular}{|c|c|c|c|}
\hline \multirow{2}{*}{$\begin{array}{c}\text { Pression de } \\
\text { référence (Pa) }\end{array}$} & \multicolumn{2}{|c|}{ Incertitude } & \multirow{2}{*}{ Sens } \\
\cline { 2 - 3 } & Absolue (Pa) & Relative (\%) & \\
\hline 0,00 & 0,08 & - & \\
\hline 30,40 & 0,22 & 0,72 & \\
\hline 49,99 & 0,27 & 0,54 & \multirow{3}{*}{ Montée } \\
\hline 101,18 & 0,40 & 0,40 & \\
\hline 150,18 & 0,65 & 0,43 & \\
\hline 199,53 & 0,70 & 0,35 & \\
\hline 151,85 & 0,65 & 0,43 & \\
\hline 101,94 & 0,40 & 0,39 & \\
\hline 51,14 & 0,27 & 0,53 & \multirow{2}{*}{ Descente } \\
\hline 30,52 & 0,22 & 0,72 & \\
\hline 0,00 & 0,08 & - & \\
\hline
\end{tabular}

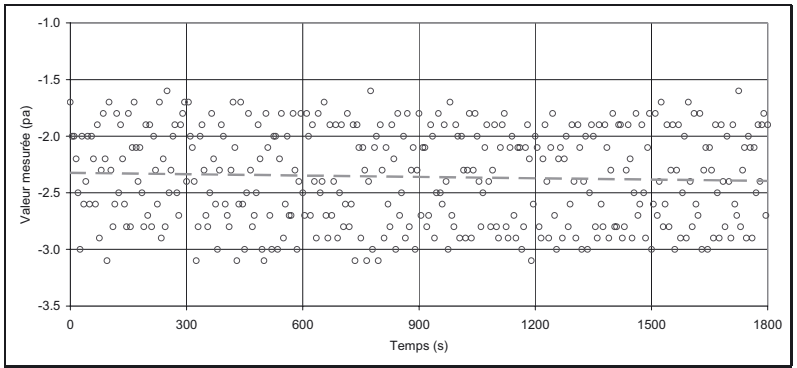

Fig. 3. - Bruit et dérive du capteur de pression.

\section{Mesure de la pression différentielle}

Un capteur de pression différentielle $(\Delta P)$ est placé dans la conduite, de part et d'autre d'un diaphragme. La perte de charge créée par ce diaphragme dépendant du débit des effluents, la mesure de $\Delta P$ permet le calcul du débit d'extraction.

Cette valeur est par ailleurs inversement proportionnelle à la température des effluents. Elle est comprise entre environ $40 \mathrm{~Pa}$ et $150 \mathrm{~Pa}$. Le certificat d'étalonnage du capteur de pression a permis d'établir l'incertitude sur $\Delta P$ (tableau 4). L'incertitude relative maximale est observée pour les valeurs les plus faibles. L'incertitude absolue du capteur peut par ailleurs être modélisée.

Les valeurs les plus faibles, de l'ordre de $40 \mathrm{~Pa}$, correspondent aux valeurs les plus fortes de dégagements de chaleur. Afin de majorer l'incertitude due à ce capteur, une valeur de $1 \%$ relatif sera utilisée dans le calcul d'incertitude.

Le bruit de fond du capteur est mesuré sans extraction, afin de s'affranchir de la rotation des pales de l'extracteur et du battement de la flamme. Les mesures réalisées sont exploitées de manière similaire à l'analyseur d'oxygène, voir paragraphe 10.1.6 de la norme ISO 5660-1 [6]. Ces mesures sont présentées à la figure 3. Elles donnent un bruit du capteur de pression efficace $u(p)=0,42 \mathrm{~Pa}$ et une dérive de 0,07 $\mathrm{Pa}$. Lors des essais, le «bruit » observé est nettement plus important. Néanmoins, ce bruit apparent correspond à la physique, car il traduit les battements de la flamme, et donc les variations locales de dégagement de chaleur. 


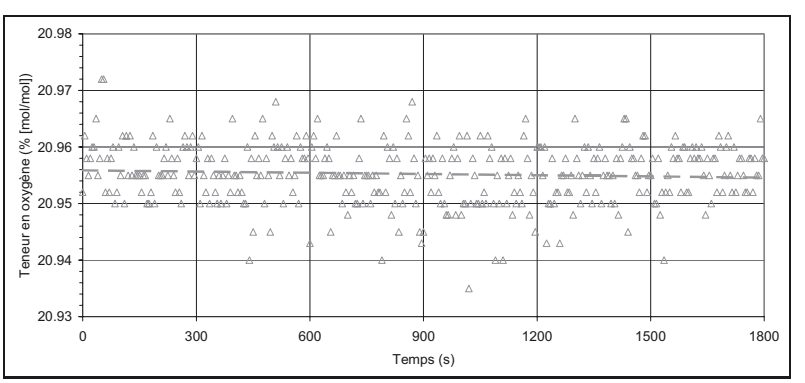

Fig. 4. - Bruit et dérive de l'analyse d'oxygène.

À chaque niveau de pression mesuré, l'incertitude type sur $\Delta P$ peut ainsi être donnée par la formule :

$$
\begin{aligned}
& u(\Delta P)= \\
& \sqrt{\left(\frac{0,01}{2} \times \Delta P\right)^{2}+(u(p))^{2}+\left(\text { dérive }_{\text {capteur pression }}\right)^{2}} .
\end{aligned}
$$

\section{Température du diaphragme}

La température $T_{\mathrm{e}}$ est mesurée sur le diaphragme à l'aide d'un thermocouple type K. Cette température varie entre a température ambiante et environ $300{ }^{\circ} \mathrm{C}$. En l'absence de données supplémentaires, l'incertitude sur cette mesure est supposée suivre une loi uniforme avec $U\left(T_{\mathrm{e}}\right)=2,5 \mathrm{~K}$ Cette valeur est l'erreur maximum tolérée pour des températures inférieures à $300{ }^{\circ} \mathrm{C}$ et des thermocouples de type $\mathrm{K}[15,16]$.

L'estimation de l'incertitude-type sur $T_{\mathrm{e}}$ est alors d'environ 1,44 K, comme donné par la relation (5).

$$
u\left(T_{\mathrm{e}}\right)=\frac{2,5}{\sqrt{3}} .
$$

\section{Mesure de la teneur molaire initiale en oxygène $x_{\mathrm{O}_{2}}^{0}$}

Cette valeur est de l'ordre de $0,2095 \mathrm{~mol} \cdot \mathrm{mol}^{-1}$ pour de l'air sec. Le bruit efficace de l'analyseur est évalué sur cette valeur. Le paragraphe 6.11 de la norme ISO 5660-1 spécifie la valeur limite de bruit de fond efficace pour l'analyseur d'oxygène comme étant de $50 \times$ $10^{-6} \mathrm{~mol} \cdot \mathrm{mol}^{-1}$. Une mesure sur $30 \mathrm{~min}$ avec un pas d'échantillonnage de $5 \mathrm{~s}$ a été réalisée et fait l'objet de la figure 4. L'analyse des résultats montre que les valeurs prennent des niveaux discrétisés. Ces niveaux proviennent de la numérisation du signal par la centrale d'acquisition. En effet, celle-ci travaille en 12 bits, c'est-à-dire qu'elle a une résolution en tension de $2^{12}=$ 4096 pas par gamme de travail.

Le bruit efficace $u\left(\mathrm{O}_{2}\right)$ trouvé est de $53 \times$ $10^{-6} \mathrm{~mol} \cdot \mathrm{mol}^{-1}$, ce qui est supérieur aux indications de la norme ISO 5660-1. Néanmoins, cette valeur ne s'écarte que de $6 \%$ de la valeur limite théorique.

L'influence de ce bruit de fond sur la mesure a ainsi été déterminée pour trois valeurs de dégagement de chaleur produits à l'aide d'une flamme de méthane $(1 \mathrm{~kW}, 3 \mathrm{~kW}$ et $5 \mathrm{~kW})$. Cette vérification consiste à regarder l'influence effective du bruit de fond sur la mesure. Les résultats montrent que pour les faibles diminutions d'oxygène, et des teneurs d'oxygène de l'ordre de $20,5 \%$, l'impact du bruit de fond sur le résultat peut être considérable. L'incertitude correspondant aux faibles valeurs de dégagement de chaleur a probablement pour origine la faible diminution d'oxygène correspondante, trop proche du bruit de fond. Dans ce cas, une réduction du débit des effluents doit permettre de gagner en sensibilité, mais les conditions de l'expérience s'écartent alors de celles de la norme. Cette possibilité est à l'étude au sein de l'ISO TC92/SC1/WG5 en charge du « cone calorimètre ».

La mesure de la concentration initiale en oxygène $x_{\mathrm{O}_{2}}^{0}$ est obtenue en début d'essai à partir de la moyenne des valeurs durant $60 \mathrm{~s}$ dans l'air avec un pas de $5 \mathrm{~s}$, c'està-dire sur douze valeurs. Le bruit correspondant est donc moyenné pour ces douze mesures. La justesse n'est pas considérée, car c'est la variation d'oxygène qui est importante dans la mesure.

L'estimation de l'incertitude type sur $x_{\mathrm{O}_{2}}^{0}$ est alors :

$$
u\left(x_{\mathrm{O}_{2}}^{0}\right)=\frac{u\left(\mathrm{O}_{2}\right)}{\sqrt{12}} .
$$

\section{Mesure de la teneur molaire en oxygène}

Lors de l'essai, la teneur molaire en oxygène des effluents est comprise entre 20,95\% et environ 18,00\%. Chaque mesure individuelle est impactée de l'erreur liée au bruit et à la chaîne d'acquisition, ainsi que de la justesse et de la fidélité de l'analyseur d'oxygène. L'analyseur a ainsi été vérifié à l'aide d'un système de dilution et d'un analyseur de référence sur le domaine [21-17] \%.

La valeur utilisée dans le calcul d'incertitude est obtenue à l'aide de l'écart maximum $e_{\max }$ entre la valeur théorique après étalonnage et la valeur mesurée dans la gamme de travail, considérant une distribution uniforme.

$$
u_{\text {analyse }}=\frac{e_{\max }}{\sqrt{3}}
$$

L'estimation de l'incertitude type sur $x_{\mathrm{O}_{2}}$ est alors

$$
u\left(x_{\mathrm{O}_{2}}\right)=\sqrt{\left(u\left(\mathrm{O}_{2}\right)\right)^{2}+\text { dérive }{ }^{2}+u_{\text {analyse }}^{2}} .
$$

\subsubsection{Acquisition et traitement des données}

L'acquisition est automatisée à partir d'un ordinateur et d'une interface d'acquisition spécifique travaillant sur 12 bits. Devant les incertitudes de détermination d'un paramètre comme le dégagement de chaleur, l'incertitude de numérisation des données est considérée comme 
incluse dans les bruits efficaces du capteur de pression $u(p)$ et de l'analyseur de dioxygène $u\left(\mathrm{O}_{2}\right)$.

\subsubsection{Considérations sur la justesse et la fidélité de la mesure}

\subsubsection{Justesse et limitation du biais}

La limitation du biais peut se faire à partir d'actions métrologiques élémentaires : la métrologie du four et celle des autres capteurs permettant la mesure du dégagement de chaleur : débit et analyseurs de gaz. La vérification du four peut être faite par 'une mesure à l'aide d'un fluxmètre étalon; la vérification des autres capteurs influents sur la mesure du dégagement de chaleur peut se faire à partir d'un matériau de référence. De plus, lors de l'étalonnage de l'appareil, le biais est limité à partir d'une combustion de référence au méthane effectuée chaque journée d'essai.

Cette limitation du biais ne prend pas en compte les effets liés au facteur de Thornton et au facteur d'expansion des gaz.

\subsubsection{Fidélité de la mesure}

De nombreux paramètres influent sur la fidélité de la mesure. L'influence de ces paramètres ne peut pas être limitée par des actions métrologiques spécifiques, car elle a de nombreuses origines ne dépendant pas toutes de l'appareillage. Elle est évaluée par analyse statistique des résultats d'essais et est présentée dans les paragraphes suivants.

\subsection{Incertitude sur le facteur $C$}

Le « facteur $C$ » de l'équation (2) est le coefficient d'étalonnage du diaphragme. Physiquement, ce facteur représente le coefficient de contraction de la veine d'air au niveau du diaphragme. Il ne dépend que de la géométrie de celui-ci et de la température. Ce paramètre est difficile à mesurer. De ce fait, la méthode de détermination est basée sur l'utilisation d'une flamme de puissance connue et une résolution inverse de l'équation (2).

Une étude a été menée sur la détermination de l'incertitude sur le « facteur $C$ » en 2004 au LNE [17]. Elle a montré que l'incertitude type relative sur ce facteur était de 2,92\%. D'autres estimations ont été proposées par Zhao et Dembsey [10], mais elles ne sont pas satisfaisantes car elles confondent incertitude et écart maximum toléré. Enright et Fleischmann [18], puis Guillaume et. coll. [19] ont proposé des approches alternatives pour la détermination du « facteur $C$ », mais celles-ci ne sont pas appliquées.

Du fait de la méthode de détermination du «facteur $C$ », des covariances négatives peuvent apparaitre dans le calcul de l'incertitude sur le dégagement de chaleur. Cela signifie que l'incertitude est majorée, car nous négligeons ces covariances dans le calcul.

\subsection{Incertitude sur la mesure}

La méthode des dérivées partielles permet d'écrire :

$$
\begin{aligned}
u(\dot{q}(t))^{2}= & \left(\frac{\partial \dot{q}(t)}{\partial C}\right)^{2} u(C)^{2}+\left(\frac{\partial \dot{q}(t)}{\partial \Delta P}\right)^{2} u(\Delta P)^{2} \\
& +\left(\frac{\partial \dot{q}(t)}{\partial T_{\mathrm{e}}}\right)^{2} u\left(T_{\mathrm{e}}\right)^{2}+\left(\frac{\partial \dot{q}(t)}{\partial x_{\mathrm{O}_{2}}^{0}}\right)^{2} u\left(x_{\mathrm{O}_{2}}^{0}\right)^{2} \\
& +\left(\frac{\partial \dot{q}(t)}{\partial x_{\mathrm{O}_{2}}}\right)^{2} u\left(x_{\mathrm{O}_{2}}\right)^{2}
\end{aligned}
$$

avec :

$$
\begin{aligned}
& \frac{\partial \dot{q}(t)}{\partial C}=\frac{1,1 E \times \sqrt{\frac{\Delta P}{T_{\mathrm{e}}}} \times\left(x_{\mathrm{O}_{2}}-x_{\mathrm{O}_{2}}^{0}\right)}{1,5 x_{\mathrm{O}_{2}}-1,105} \\
& \text { et } \frac{\partial \dot{q}(t)}{\partial C}=\frac{\dot{q}(t)}{C} \\
& \frac{\partial \dot{q}(t)}{\partial \Delta P}=\frac{1,1 C \cdot E \times\left(x_{\mathrm{O}_{2}}-x_{\mathrm{O}_{2}}^{0}\right)}{2 \sqrt{\frac{\Delta P}{T_{\mathrm{e}}}} \times\left(1,5 x_{\mathrm{O}_{2}}-1,105\right) \times T \mathrm{e}} \\
& \frac{\partial \dot{q}(t)}{\partial T_{\mathrm{e}}}=\frac{-1,1 C \cdot E \cdot \Delta P \times\left(x_{\mathrm{O}_{2}}-x_{\mathrm{O}_{2}}^{0}\right)}{2 \sqrt{\frac{\Delta P}{T_{\mathrm{e}}}} \times T_{\mathrm{e}}^{2} \times\left(1,5 x_{\mathrm{O}_{2}}-1,105\right)} \\
& \frac{\partial \dot{q}(t)}{\partial x_{\mathrm{O}_{2}}^{0}}=\frac{-1,1 C \cdot E \times \sqrt{\frac{\Delta P}{T_{\mathrm{e}}}}}{\left(1,5 \cdot x_{\mathrm{O}_{2}}-1,105\right)} \\
& \frac{\partial \dot{q}(t)}{\partial x_{\mathrm{O}_{2}}}=\frac{-1,1 C \cdot E \times \sqrt{\frac{\Delta P}{T_{\mathrm{e}}}} \times\left(1,105-1,5 x_{\mathrm{O}_{2}}^{0}\right)}{\left(1,5 x_{\mathrm{O}_{2}}-1,105\right)^{2}} \\
& \frac{\partial \dot{q}(t)}{\partial E}=1,10 C \times \sqrt{\frac{\Delta P}{T_{\mathrm{e}}}} \times \frac{x_{\mathrm{O}_{2}}^{0}-x_{\mathrm{O}_{2}}}{1,105-1,5 x_{\mathrm{O}_{2}}} \\
& \frac{u(\dot{q}(t))^{2}}{(\dot{q}(t))^{2}}=\frac{u(C)^{2}}{C^{2}}+\frac{u(\Delta P)^{2}}{\Delta P^{2}}+\frac{u\left(T_{\mathrm{e}}\right)^{2}}{\left(T_{\mathrm{e}}\right)^{2}} \\
& +\frac{u\left(x_{\mathrm{O}_{2}}^{0}\right)^{2}}{\left(x_{\mathrm{O}_{2}}^{0}\right)^{2}}+\frac{u\left(x_{\mathrm{O}_{2}}\right)^{2}}{\left(x_{\mathrm{O}_{2}}\right)^{2}} \text {. }
\end{aligned}
$$

\section{Autres mesurandes}

\subsection{Dégagement de chaleur maximum}

Pour chaque essai individuel, l'incertitude sur la valeur maximum est calculée de la même manière que pour toutes les valeurs. Pour une série de $N$ essais, l'effet matériau est pris en compte à partir de la dispersion des mesures. Dans ce cas, l'incertitude sur la moyenne des valeurs maximales de dégagement de chaleur est donnée par:

$$
u_{\dot{q}_{\max }}=\sqrt{\frac{\sigma_{\text {dispersion }}^{2}}{N}+\sum_{i=1}^{N} \sigma_{\dot{q}_{\max }}^{2}} .
$$




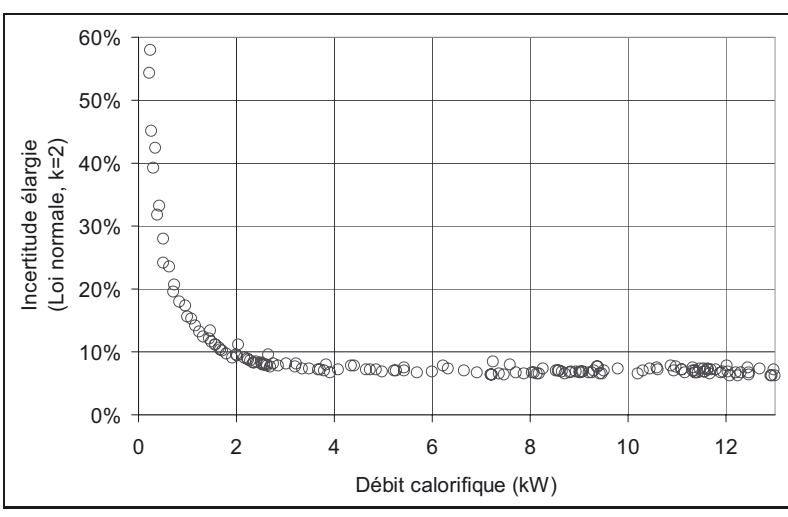

Fig. 5. - Détermination de l'incertitude sur la mesure du dégagement de chaleur.

Si la valeur est exprimée par rapport à la surface d'exposition telle que définie par le porte-éprouvette normalisé, une composante d'incertitude supplémentaire s'ajoute. Les tolérances de la fenêtre d'exposition donnent un carré de côté $L_{\text {porte éprouvette }}=(94,0 \pm 0,5) \mathrm{mm}$. En faisant l'hypothèse d'une loi normale pour les tolérances d'usinage, l'aire de la fenêtre d'exposition est $A=8836 \mathrm{~mm}^{2}$. L'incertitude réduite sur l'aire est de :

$$
u_{A}=\sqrt{2 L_{\text {porte éprouette }} \times\left(\frac{0,0005}{2}\right)^{2}}=108 \mathrm{~mm}^{2} .
$$

Le dégagement de chaleur étant ramené à l'unité de surface, les incertitudes se propagent en valeurs relatives. Donc, l'incertitude relative sur les valeurs exprimées par rapport à la surface est la somme des incertitudes relatives sur l'aire et sur le dégagement de chaleur moyen.

\subsection{Energie totale dégagée}

L'énergie dégagée par unité de surface à chaque instant $\dot{Q}_{i}^{\prime \prime}$ est donnée par intégration de la courbe de dégagement de chaleur suivant la méthode des trapèzes :

$$
\dot{Q}_{i}^{\prime \prime}=\sum_{1}^{i} \frac{\dot{q}_{i-1}^{\prime \prime}+\dot{q}_{i}^{\prime \prime}}{2 \times\left(t_{i}-t_{i-1}\right)} .
$$

Donc, à chaque valeur de $\dot{Q}_{i}^{\prime \prime}$, l'incertitude est donnée par :

$$
u_{\dot{Q}_{i}^{\prime \prime}}=\sqrt{u_{\dot{Q}_{i-1}^{\prime \prime}}^{2}+\left(\frac{-1}{2 \times\left(t_{i-1}-t_{i}\right)}\right) \times\left(u_{\dot{q}_{i-1}^{\prime \prime}}^{2}+u_{\dot{q}_{i}^{\prime \prime}}^{2}\right)} .
$$

L'incertitude sur la mesure du temps est négligée. L'incertitude correspondant à la dernière valeur de $\dot{Q}^{\prime \prime}$ est celle sur l'énergie totale dégagée.

\section{Application aux résultats d'un essai}

\subsection{Dégagement de chaleur associé à une mesure unique}

La courbe de la figure 5 présente les résultats du calcul pour tous les points de mesure lors d'un essai unique.

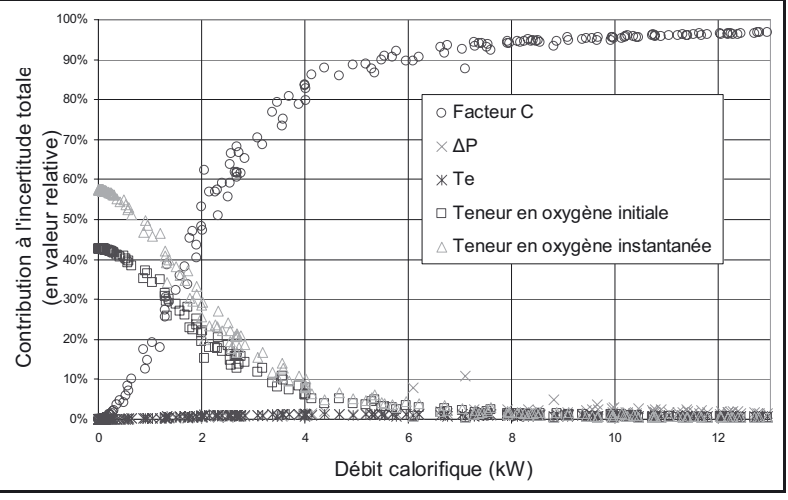

Fig. 6. - Évolution relative des différentes composantes de l'incertitude en fonction de la valeur du dégagement de chaleur.

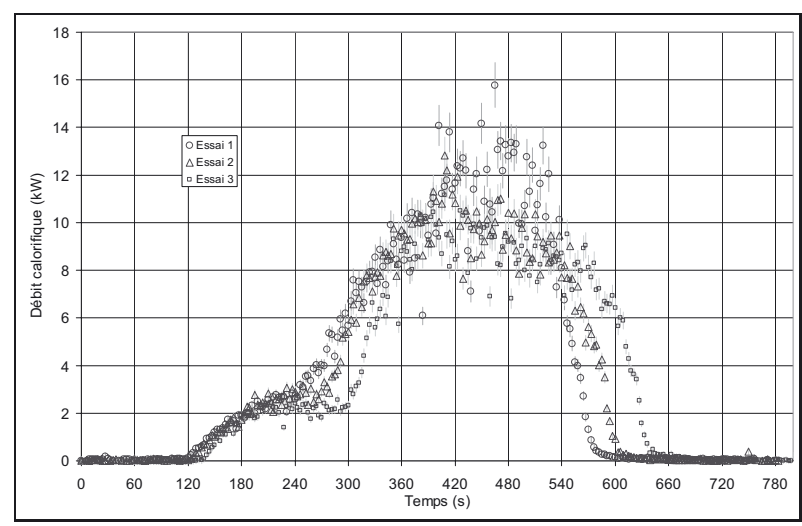

Fig. 7. - Exemple d'application à une série d'essais.

Elle est caractéristique de tous les essais uniques et ne prend pas en compte l'effet matériau. Les résultats obtenus montrent que pour des niveaux de dégagement de chaleur inférieurs à $2 \mathrm{~kW}$, l'incertitude augmente rapidement. Au-delà, l'incertitude relative tend vers un peu moins de $10 \%$.

Le calcul des différentes composantes d'incertitude est présenté à la figure 6 . Il montre que pour les faibles valeurs de dégagement de chaleur, l'incertitude provient presque exclusivement des concentrations en oxygène initiale et instantanée. Au-dessus de $1 \mathrm{~kW}$ à $2 \mathrm{~kW}$, les autres grandeurs d'influence interviennent, en particulier le coefficient $C$ qui explique plus de $90 \%$ de l'incertitude au-delà de $6 \mathrm{~kW}$.

\subsection{Série d'essais sur un matériau}

L'exemple présenté à la figure 7 montre trois répétitions d'essais sur le même matériau. Les incertitudes figurent sur le graphique. Les résultats sur la valeur de dégagement de chaleur maximum et sur la chaleur totale dégagée sont détaillés dans le tableau 5.

Dans cet exemple, les écarts entre les courbes sont supérieurs aux incertitudes. Cela s'explique par l'effet matériau, plus important que l'incertitude sur la mesure individuelle. L'incertitude sur chacune des valeurs de dégagement de chaleur maximum est de l'ordre de $12 \%$ 
Tableau 5

Exemple de résultats pour une série d'essais.

\begin{tabular}{|c|c|c|c|c|}
\hline & \multirow{2}{*}{$\begin{array}{c}\dot{q}_{\max } \\
(\mathbf{e n ~ k W )})\end{array}$} & \multicolumn{2}{|c|}{$U\left(\dot{q}_{\max }\right)$} \\
\hline & & & (en kW) & (en \%) \\
\hline & Essai 1 & 15,78 & 1,87 & 11,8 \\
\hline & Essai 2 & 12,83 & 1,53 & 11,9 \\
\hline & Essai 3 & 11,16 & 1,33 & 11,9 \\
\hline \multirow{2}{*}{$\overline{\dot{q}}_{\max }$} & (en kW) & 13,26 & 3,13 & 23,6 \\
\hline & $\left(\mathrm{en} \mathrm{kW} \cdot \mathrm{m}^{-2}\right)$ & 1500,5 & 357,3 & 23,8 \\
\hline & & $\dot{Q}$ & \multicolumn{2}{|c|}{$U(\dot{Q})$} \\
\hline & & (en kJ) & (en kJ) & $($ en \%) \\
\hline & Essai 1 & 3088,0 & 12,8 & 0,4 \\
\hline & Essai 2 & 2950,8 & 11,9 & 0,4 \\
\hline & Essai 3 & 2876,2 & 11,4 & 0,4 \\
\hline \multirow{2}{*}{$\bar{Q}$} & $(\mathrm{en} \mathrm{kJ})$ & 2972 & 125 & 4,2 \\
\hline & $\left(\mathrm{en} \mathrm{MJ} \cdot \mathrm{m}^{-2}\right)$ & 336,3 & 17,0 & 5,1 \\
\hline
\end{tabular}

et celle sur la moyenne de $24 \%$. L'incertitude de mesure contribue donc fortement à celle sur la moyenne. L'écart type de dispersion des mesures est de 2,34 kW (20\%).

L'incertitude sur l'énergie totale dégagée est nettement plus faible, du fait de l'effet «filtre » de l'intégration. Elle provient presque uniquement de l'écart type expérimental entre les trois essais $(107 \mathrm{~kJ})$ (effet matériau).

\section{Références}

[1] BABRAUSKAS V., "Development of the cone calorimeter - A bench-scale heat release rate apparatus based on Oxygen consumption", NBSIR82-2611, National Bureau of Standard, Gaithersburg, 1982, USA.

[2] BABRAUSKAS V., "Development of the cone calorimeter A bench-scale heat release rate apparatus based on oxygen consumption", Fire and Materials, 1984, 8, 81-95.

[3] Hugett C., "Estimation of rate of heat release by means of oxygen consumption measurements", Fire and Materials., 1980, 12, 2, 61-65.

[4] JANSSENS M.L., "Measuring rate of heat release by oxygen consumption", Fire Technology, 1991, 27, 234-249.

Article reçu le 10 janvier 2011, version révisée reçue le 26 février 2013.
[5] THORNTON W., "The relation of Oxygen to the heat of combustion of organic compounds", Philosophical Magazine and Journal of Science, 1917, 33, 196-203.

[6] ISO 5660-1:2002, "Reaction-to-fire tests - Heat release, smoke production and mass loss rate - Part 1: heat release rate (cone calorimeter method)".

[7] Guillaume E., « Mesure du débit calorifique par analyse de gaz. Synthèse bibliographique », Document G020284/CEMATE/3, Laboratoire national de métrologie et d'essais, oct. 2006 (non publié, disponible sur demande auprès de l'auteur).

[8] EnRight P.A. et Fleischmann C.M., "Uncertainty of heat release rate calculation of the ISO5660-1 cone calorimeter standard test method", Fire Technology, 1999, 35, $2,153-169$.

[9] ZHAO L., "Bench scale apparatus measurement uncertainty and uncertainty effects on measurement of fire characteristics of material system", $\mathrm{PhD}$ Thesis, Worcester Polytechnic Institute, mai 2005.

[10] ZhAO L. et DEMBSEY N.A., "Measurement uncertainty analysis for calorimetry apparatuses", Fire and Materials, 2008, 32, 1, 1-26.

[11] BRoHEz S., "Comments to the paper uncertainty of heat release rate calculation of the ISO5660-1 cone calorimeter standard test method", Fire Technology, 2009, 45, 4, 381384.

[12] "NATO STANAG 4602, Fire assessment of materials", 2004.

[13] "NATO Reaction to fire tests for materials. Heat released", AFAP-5 Edition 3, juill. 2010. http://www.nato.int/docu/ stanag/afap/AFAP-5\%20Ed3.pdf.

[14] ISO/IEC Guide 98-3:2008, "Uncertainty of measurement - Part 3: Guide to the expression of uncertainty in measurement (GUM)", 1995.

[15] IEC 60584-2:1982. "Thermocouples - Part 2: tolerances".

[16] IEC 60584-2/A1:1989, "Amendment 1 to publication IEC 584-2-1982".

[17] «Calcul d'incertitude sur le facteur $C$ », Rapport et procédure interne LNE, Document 672H0899_08, Laboratoire national de métrologie et d'essais, 2004 (non publié).

[18] EnRight P.A. et Fleischmann C.M., "Cone calorimeter - An alternative calibration constant calculation", Fire and Materials, 24, 4, 2000, 187-193.

[19] Guillaume E., Marquis D. et Saragoza L., "Calibration of flow rate in cone calorimeter tests", Fire and Materials, à paraître en 2013, DOI: 10.1002/fam. 2174. 